International Journal of Pure and Applied Mathematics

Volume $91 \quad$ No. $3 \quad 2014,389-394$

ISSN: 1311-8080 (printed version); ISSN: 1314-3395 (on-line version)

url: http://www.ijpam.eu

doi: http://dx.doi.org/10.12732/ijpam.v91i3.12

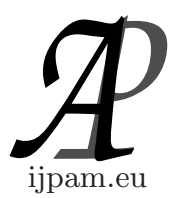

\title{
OPEN RANK (OR WIDERANK) FOR REDUCIBLE PROJECTIVE SETS: A CLASSIFICATION OF AN EXTREMAL CASE
}

\author{
E. Ballico \\ Department of Mathematics \\ University of Trento \\ 38123 Povo (Trento) - Via Sommarive, 14, ITALY
}

\begin{abstract}
Let $X \subset \mathbb{P}^{r}, r \geq 2$, be a reduced projective set such that $\langle X\rangle=\mathbb{P}^{r}$, where \langle\rangle denote the linear span of $X$. For any $P \in \mathbb{P}^{r}$ the $X$-widerank $w_{X}(P)$ of $P$ is the minimal integer $t>0$ such that for each closed set $B \subset X$ containing no irreducible component of $X$ there is $S \subset X \backslash B$ with $P \in\langle S\rangle$. Here we classify all $(r, X)$ such that $w_{X}(P) \geq r+1$ for a general $P(r$ is odd and $X$ is the union of $(r+1) / 2$ linearly independent lines). We give conditions on $X$ which imply that $w_{X}(P) \leq r\left(\right.$ or $\left.w_{X}(P) \leq r+1-\operatorname{dim}(X)\right)$ for every $P \in \mathbb{P}^{r} \backslash X$.
\end{abstract}

AMS Subject Classification: 14N05, 14Q05, 15A69

Key Words: widerank, open rank, symmetric tensor rank, reducible varieties, lines, strange variety

\section{The Statements}

For each set $B \subseteq \mathbb{P}^{r}$ let $\langle B\rangle$ denote its linear span.

Let $X \subset \mathbb{P}^{r}, r \geq 2$, be a reduced projective set such that $\langle X\rangle=\mathbb{P}^{r}$. For each $P \in \mathbb{P}^{r}$ the $X$-rank $r_{X}(P)$ of $P$ is the minimal cardinality of a set $S \subset X$ such that $P \in\langle S\rangle$. Now assume that no component of $X$ is a single point. The $X$-widerank $w_{X}(P)$ of $P$ is the minimal integer $t>0$ such that for each closed set $B \subset X$ containing no irreducible component of $X$ there is $S \subset X \backslash B$ with

Received: December 20, 2013

(c) 2014 Academic Publications, Ltd. url: www.acadpubl.eu 
$P \in\langle B\rangle$ and $\sharp(S) \leq t$. We have $w_{X}(P) \leq r+1$ for all $P$ and all $X$ and there are known several pairs $(X, P)$ with $w_{X}(P)=r+1$ (e.g., if $X$ is a rational normal curve, then take all $P \in X)$. A different problem is the generic $X$-widerank, i.e. the $X$-widerank of a general $P \in \mathbb{P}^{r}$. In this note we prove the following results.

Theorem 1. There is a non-empty open subset $U$ of $\mathbb{P}^{r}$ such that $w_{X}(P)=$ $r+1$ for all $P \in U$ if and only if $r$ is odd and $X$ is a disjoint union of $(r+1) / 2$ lines spanning $\mathbb{P}^{r}$.

All $X$ 's as in Theorem 1 are projectively equivalent. For these $X$ 's the set of all $P \in \mathbb{P}^{r}$ with $w_{X}(P)=r+1$ is the set of all $P$ not contained in one of the $(r+1) / 2$ codimension 2 linear subspaces of $\mathbb{P}^{r}$ spanned by $(r-1) / 2$ lines of $X$.

Proposition 1. Assume the existence of an irreducible component $Y$ of $X$ such that $\operatorname{deg}(Y)>1$. Then $w_{X}(P) \leq r+1-\operatorname{dim}(Y)$ for a general $P \in \mathbb{P}^{r}$.

If $X$ is a Veronese embedding of $\mathbb{P}^{m}$, then the definition of $w_{X}(P)$ is related to the open rank or $(P)$ defined in [2]: when $\operatorname{or}(P)$ is defined, then $\operatorname{or}(P)=$ $w_{X}(P)$, but $\operatorname{or}(P)$ is defined only for points associated to forms depending on all $m+1$ homogeneous variables.

We work over an algebraically closed field $\mathbb{K}$ with arbitrary characteristic.

\section{The Proofs}

To prove Proposition 1 we need the following classical definition. Fix any integral variety $Y \subset \mathbb{P}^{r}$ with positive dimension and $P \in \mathbb{P}^{r}$. $P$ is said to be a strange point of $Y$ if every tangent space to $Y$ at a smooth point of $Y$ contains $P$. Let $\Sigma(Y)$ denote the set of all strange points of $Y$. In characteristic zero $\Sigma(Y) \neq \emptyset$ if and only if $Y$ is a cone and in this case $\Sigma(Y)$ is the vertex of $Y$. In positive characteristic $\Sigma(Y)$ is a linear space with dimension at most $\operatorname{dim}(Y)$ (unless $Y$ is a linear space).

Lemma 1. Assume that $X$ has an irreducible component $Y$ which is a linear space of dimension $m \geq 2$. Then $r_{X}(P) \leq r+1-m$ for all $P \in \mathbb{P}^{r}$ and $w_{X}(P) \leq r+2-m$ for all $P \in \mathbb{P}^{r} \backslash Y$.

Proof. Fix $P \in \mathbb{P}^{r}$. If $P \in Y$, then $r_{X}(P)=1$. Now assume $P \notin Y$ and take a closed subset $B \subset X$ containing no irreducible components of $X$. Since $\mathbb{P}^{r} \backslash Y \neq \emptyset$, we have $r>m$. Since $\langle Y\rangle=Y$ and $X$ spans $\mathbb{P}^{r}$, the set $X \backslash(Y \cup B)$ spans a linear space $M$ such that $\langle M \cup Y\rangle=\mathbb{P}^{r}$. Hence there are $S \subset X \backslash(Y \cup B)$ and $O \in Y$ such that $\sharp(S)=r-m$ and $P \in\langle S \cup\{O\}\rangle$. Since 
$B \cap Y$ is a proper closed subset of $Y$ there is a line $L \subset Y$ such that $O \in L$ and $L \cap B$ is finite. Take $E \subset L \backslash L \cap B$ such that $\sharp(E)=2$. Since $\langle E\rangle=L$, we have $O \in\langle E\rangle$. Hence $P \in\langle S \cup E\rangle$. Since $S \cup E \subset X \backslash B$, we get $w_{X}(P) \leq r+2-m$. Since $S \cup\{O\} \subset X$, we get $r_{X}(P) \leq r+1-m$.

Lemma 2. Let $Y \subsetneq \mathbb{P}^{n}$ be an integral and non-degenerate variety. Then $w_{Y}(P) \leq n+1-\operatorname{dim}(Y)$ for all $P \in \mathbb{P}^{r} \backslash(Y \cup \Sigma(Y))$.

Proof. Fix $P \in \mathbb{P}^{r} \backslash(Y \cup \Sigma(Y))$ and a closed subset $B \subsetneq Y$. Since $P \notin$ $\Sigma(Y)$, a characteristic free version of Bertini's theorem gives that for a general linear subspace $M \subset \mathbb{P}^{n}$ with $P \in M$ and $\operatorname{dim}(M)=n-\operatorname{dim}(Y)$ the scheme $M \cap Y$ is a reduced set spanning $M$ ([1], proof of Theorem 1 up to line 12 of page 6). Hence there is $S \subset M \cap Y$ such that $\sharp(S)=n+1-\operatorname{dim}(Y)$ and $P \in\langle S\rangle$. Since $P \notin Y, \operatorname{dim}(B) \leq \operatorname{dim}(Y)-1$ and $M$ is a general linear space of dimension $n-\operatorname{dim}(Y)$ containing $P$, we have $M \cap B=\emptyset$. Hence $S \subset Y \backslash B$.

Proposition 2. Let $X \subset \mathbb{P}^{r}$ be equidimensional and non-degenerate with dimension $m>0$. Assume that $X$ is connected in codimension $m-1$, i.e. it is connected if $m=1, X \backslash A$ is connected for all closed sets $A \subset X$ with $\operatorname{dim}(A) \leq m-2$ if $m \geq 2$. Let $\Sigma$ be the union of the strange sets of the irreducible components of $X$. Then $w_{X}(P) \leq r+1-m$ for all $P \in \mathbb{P}^{r} \backslash(X \cup \Sigma)$.

Proof. Apply $m-1$ times [1], proof of Theorem 1 up to line 12 of page 6, and the proof of Lemma 2. In the case $m=1$ and $\operatorname{char}(\mathbb{K})=0$, then this is the proof of [2], Lemma 11.

Proof of Proposition 1. Fix $P \in \mathbb{P}^{r} \backslash(Y \cup \Sigma(Y))$ and take a closed subset $B \subset X$ containing no irreducible component of $X$. Set $e:=\operatorname{dim}(\langle Y\rangle)$. Since $\operatorname{deg}(Y)>1$, we have $e>\operatorname{dim}(Y)$. If $e=r$, then we may apply Lemma 2, because $w_{Y}(P)$ is defined and $w_{X}(P) \leq w_{Y}(P)$. Hence we may assume $r>e$. Since $X$ spans $\mathbb{P}^{r}$ there is $S \subset X \backslash(Y \cup B)$ such that $\sharp(S)=r-e$ and $\langle Y\rangle \cup \mathbb{P}^{r}$. Hence there is $O \in\langle Y\rangle$ such that $P \in\langle\{O\} \cup S\rangle$. If $O \notin Y \cup \Sigma(Y)$, then there is $E \subset Y \backslash Y \cap B)$ such that $\sharp(E) \leq e-\operatorname{dim}(Y)$ and $O \in\langle E\rangle$. In this case the set $E \cup S \subset X \backslash B$ shows that $w_{X}(P) \leq r+1-\operatorname{dim}(Y)$. For a general $P \in \mathbb{P}^{r}$ we may find $S$ and $O$ with $O \notin Y \cup \Sigma(Y)$. Hence $w_{X}(P) \leq r+1-\operatorname{dim}(Y)$ for a general $P \in \mathbb{P}^{r}$.

Proof of Theorem 1. We first check the "if " part. Take $r=2 k-1$, $k \geq 2$, and write $X=L_{1} \sqcup \cdots \sqcup L_{k}$ with each $L_{i}$ a line and $\langle X\rangle=\mathbb{P}^{r}$. Any two $X$ 's as above are projectively equivalent. Let $U$ be the set of all $P \in \mathbb{P}^{r}$ not contained in one of the $k$ codimension 2 linear subspaces of $\mathbb{P}^{r}$ spanned by $k-1$ 
lines of $X$. Fix any $P \in U$. Linear algebra teach us that $r_{X}(P)=k$, that there is a unique set $S \subset X$ with $\sharp(S)=k$ and $P \in\langle S\rangle$ and that $\sharp\left(S \cap L_{i}\right)=1$ for all $i$. Set $P_{i}:=S \cap L_{i}$. Moreover, for each $i \in\{1, \ldots, k\} P_{i}$ is the only $O \in L_{i}$ such that $P \in\left\langle\{O\} \cup\left(X \backslash L_{i}\right)\right.$. Take any finite $B \subset X$ with $B \supseteq S$ and any $A \subset X \backslash B$ with $\sharp(A)<2 k$. There is $i \in\{1, \ldots, k\}$ such that $\sharp\left(A \cap L_{i}\right) \leq 1$. Take $F \supseteq A$ such that $F \subset X \backslash S, \sharp\left(F \cap L_{i}\right)=1$ and $\sharp\left(F \cap L_{j}\right) \geq 2$. Write $\{O\}:=F \cap L_{i}$. Since $F \subset\{O\} \cup\left(X \backslash L_{i}\right)$ and $O \neq P_{i}$, we have $P \notin\langle F\rangle$. Hence $w_{X}(P) \geq 2 k$. Since the inequality $w_{X}(P) \leq r+1$ is obvious for any $X$ and $P$, we get the " if " part.

Now assume the existence of a non-empty open subset $U$ of $\mathbb{P}^{r}$ such that $w_{X}(P) \geq r+1$ for all $P \in U$. By Proposition 1 and Lemma 1 we may assume that each irreducible component of $X$ is a line. Taking a smaller $U$ we may assume that no $Q \in U$ is contained in a proper linear subspace of $\mathbb{P}^{r}$ spanned by some of the lines in $X$. First assume $r=2$. Since $X$ spans $\mathbb{P}^{2}$ we have $\operatorname{deg}(X) \geq 2$. In this case we immediately see that $w_{X}(P)=2$ for all $P \in \mathbb{P}^{2}$. Now assume $r>2$ and that Theorem 1 is true in $\mathbb{P}^{r-1}$. We also use induction on the integer $\operatorname{deg}(X)$. Fix an irreducible component $L$ of $X$ and call $W$ the union of the other irreducible components of $X$. Let $\ell_{L}: \mathbb{P}^{r} \backslash L \rightarrow \mathbb{P}^{r-2}$ be the linear projection from $L$.

(i) First assume $L \cap\langle W\rangle=\emptyset$. Our assumption on $U$ gives the existence of a unique $O_{P} \in\langle W\rangle$ such that $P \in\left\langle L \cup\left\{O_{P}\right\}\right\rangle$. Take $S \subset W \backslash W \cap B$ evincing $w_{W}\left(O_{P}\right)$. Take any $E \subset L \backslash L \cap B$ with $\sharp(E)=2$. Since $E \cup S \subset X \backslash B$, we get $\sharp(E \cup S) \geq w_{X}(P)=r+1$. Hence $\sharp(S) \geq r-1$. Varying $P$ in $U$ the point $O_{P}$ varies in a non-empty open subset of $\langle W\rangle$. Hence $r_{W}(O) \geq r-1$ for a a general $O \in\langle W\rangle$. The inductive assumption gives that $r=2 k-1$ is odd and that $W$ is the union of $k-1$ lines spanning $\langle W\rangle$. Hence $X$ is as in the statement of Theorem 1.

(ii) Now assume that $L \cap\langle W\rangle$ is a unique point, $Q$. Hence $\operatorname{dim}(\langle W\rangle)=$ $r-1$.

(ii1) First assume $Q \notin W$. In this case $\ell_{L}$ is defined at each point of $W$. The algebraic set $\ell_{L}(W)$ is a union of finitely many lines and it spans $\mathbb{P}^{r-2}$. For a general $P$ the point $\ell_{L}(P)$ is a general point of $\mathbb{P}^{r-2}$. Assume for the moment $w_{\ell_{L}(W)}\left(\ell_{L}(P)\right) \leq r-2$ and take $S \subset \ell_{L}(W) \backslash \ell_{L}(B \cap W)$ such that $\ell_{L}(P) \in\langle S\rangle$. Since $L \cap W=\emptyset, \ell_{L} \mid W$ is a morphism and so there is $S^{\prime} \subset W$ such that $\sharp\left(S^{\prime}\right)=\sharp(S)$ and $\ell_{L}\left(S^{\prime}\right)=S$. Since $S \cap \ell_{L}(B \backslash L \cap B)=\emptyset$ and $W \cap L=\emptyset$, we have $S^{\prime} \subset W \backslash W \cap B$. Take any $E \subset L \backslash L \cap B$ such that $\sharp(E)=2$. Since $\ell_{L}(P) \in\langle S\rangle$ and $\langle E\rangle=L$, we have $P \in\left\langle E \cup S^{\prime}\right\rangle$. Since $S^{\prime} \cup E \subset X \backslash B$, we get $w_{X}(P) \leq r$, a contradiction. Hence $w_{\ell_{L}(W)}\left(\ell_{L}(P)\right) \geq r-1$. By the inductive assumption $r-2=2 k-3$ is odd and $\ell_{L}(W)$ is a disjoint union of $k-1$ lines 
spanning $\mathbb{P}^{r-2}$. Of course, $\ell_{L}$ must send at least two components of $W$ onto the same line of $\ell_{L}(W)$, because $\langle W\rangle$ has dimension $r-1$. Take an ordering $L_{1}, \ldots, L_{k-1}$ of the lines of $\ell_{L}(W)$ and call $E_{i}$ the union of the components of $W$ whose image is $L_{i}$. Since $L_{i} \cap L_{j}=\emptyset$ for all $i \neq j$, we have $E_{i} \cap E_{j}=\emptyset$ for all $i \neq j$, each $\left\langle E_{i}\right\rangle$ is a plane containing $Q$ and $\left\langle E_{i}\right\rangle \cap\left\langle E_{j}\right\rangle=\{Q\}$ for all $i \neq j$. Since $P \in\langle X\rangle$, there is $o_{P} \in L, o_{W} \in\langle W\rangle$ and $O_{i} \in\left\langle E_{i}\right\rangle, 1 \leq i \leq k-1$, such that $P \in\left\langle\left\{o_{P}, o_{W}\right\}\right\rangle$ and $o_{W} \in\left\langle\left\{O_{1}, \ldots, O_{k-1}\right\rangle\right.$. Since $P \notin\langle W\rangle$, we have $o_{P} \neq Q$. Since $L \cap\langle W\rangle \neq \emptyset$, the $k$-ple $\left(o_{P}, o_{W}\right)$ is not unique. Indeed, for suitable $o_{W} \in\langle W\rangle$ we may take as $o_{P}$ any point of $L \backslash\{Q\}$. In particular we may take $o_{W}$ so that $o_{P} \notin B$. We fix any such a choice of $o_{W}$. Either $E_{i}$ is a line or $E_{i}$ is a non-degenerate plane curve. In both cases we have $w_{E_{i}}(O)=2$ for all $O \in\left\langle E_{i}\right\rangle$. Take $F_{i} \subset E_{i} \backslash E_{i} \cap B$ such that $\sharp\left(F_{i}\right)=2$ and $O_{i} \subset\left\langle E_{i}\right\rangle$. Set $F:=\left\{o_{P}\right\} \cup F_{1} \cup \cdots \cup F_{k-1}$. We have $\sharp(F)=r, F \subset X \backslash B$ and $P \in\langle F\rangle$.

(ii2). Now assume $Q \in W$. Hence $X$ contains two lines $L, R$ such that $L \cup R$ is a reducible conic. The case $r=2$ gives $w_{L \cup R}(O)=2$ for all $O \in\langle L \cup R\rangle$. As in the proof of Lemma 1 we get $w_{X}(O) \leq r$ for all $O \in \mathbb{P}^{r}$.

(iii) Now assume $L \subset\langle W\rangle$, i.e. $\langle W\rangle=\mathbb{P}^{r}$. Hence $r_{W}(O)$ and $w_{W}(O)$ are defined for every $O \in \mathbb{P}^{r}$. We have $w_{X}(O) \leq w_{W}(O)$. By the inductive assumption on the integer $\operatorname{deg}(X)$ we get that $r=2 k-1$ is odd and that $W$ is a disjoint union of $k$ lines. Hence $\operatorname{deg}(X)=k+1$. Taking another irreducible component $L^{\prime}$ of $X$ instead of $L$ and using steps (i), (ii1) and (iv) we get that any $k$ of the components of $X$ span $\mathbb{P}^{r}$. Let $U$ be the complement in $\mathbb{P}^{2 k-1}$ of the codimension 2 linear subspaces of $\mathbb{P}^{r}$ spanned by $k-2$ of the lines of $X$. Fix any $P \in U$ and any finite set $B \subset X$. First assume $r=3$. Let $H \subset \mathbb{P}^{3}$ be a general plane through $P$. The set $X \cap H$ is formed by 3 points spanning $H$. For general $H$ we have $B \cap H=\emptyset$. Hence $w_{X}(P) \leq 3$, a contradiction. Now assume $r \geq 5$. Fix a line $L \subset X$ and set $W:=X \backslash L$. Since any $k$ of the components of $X$ span $\mathbb{P}^{r}, \ell_{L}(W)$ is a disjoint union of $k$ lines, any $k-1$ of them spanning $\mathbb{P}^{r-2}$. Hence $r_{\ell_{L}(W)}(O) \leq r-2$ for a general $O \in \mathbb{P}^{r-2}$ by the inductive assumption. Since $P \in U$, we have $P \notin L$. Hence $\ell_{L}(P)$ is welldefined. Set $B^{\prime}:=\ell_{L}(B \backslash B \cap L)$. For general $P$ the point $\ell_{L}(P)$ is a general point of $\mathbb{P}^{r-2}$. Hence there is $S \subset \ell_{L}(W) \backslash B^{\prime}$ such that $\sharp(S) \leq r-2$ and $\ell_{L}(P) \in\langle S\rangle$. Since $\ell_{L} \mid W$ is an embedding, there is a unique $S^{\prime} \subset W$ such that $\ell_{L}\left(S^{\prime}\right)=S$. Since $S \cap B^{\prime}=\emptyset$, we have $S^{\prime} \cap B=\emptyset$. Take any $E \subset L \backslash L \cap B$ such that $\sharp(E)=2$. Since $\ell_{L}(P) \in\langle S\rangle$, we have $P \in\left\langle E \cup S^{\prime}\right\rangle$. Hence $w_{X}(P) \leq r$, a contradiction. 


\section{Acknowledgements}

The author was partially supported by MIUR and GNSAGA of INdAM (Italy).

\section{References}

[1] E. Ballico, An upper bound for the X-ranks of points of $\mathbb{P}^{n}$ in positive characteristic, Albanian J. Math., No. 15 (2011), 3-10.

[2] J. Jelisiejew, An Upper Bound for the Waring Rank of a Form, arXiv:1305.6957. 